\title{
Presaccadic target competition attenuates distraction
}

\author{
Nicholas E. DiQuattro ${ }^{1,2}$ • Joy J. Geng ${ }^{1,2}$
}

Published online: 3 February 2017

(C) The Psychonomic Society, Inc. 2017

\begin{abstract}
Although it is well known that salient nontargets can capture attention despite being task irrelevant, several studies have reported short fixation dwell times, suggesting the presence of an attentional mechanism to "rapidly reject" dissimilar distractors. Rapid rejection has been hypothesized to depend on the strong mismatch between distractor features and the target template, but it is unknown whether the presence of strong feature mismatch is sufficient, or if the presence of a target at a competing location is also necessary. Here, we investigated this question by first replicating the finding of rapid rejection for dissimilar distractors in the presence of a concurrent target (Experiment 1); manipulating the onset of the target stimulus relative to the distractor (Experiment 2); and using a saccade-contingent display to delay the target onset until after the first saccade was initiated. The results demonstrate that the speed of distractor rejection depends on the presence of target competition prior to the initiation of the first saccade, and not after the saccade. This suggests that stimulus competition for covert attention sets a "saccade priority map" that unfolds over time, resulting in faster corrective saccades to an anticipated object with higher top-down attentional priority.
\end{abstract}

Electronic supplementary material The online version of this article (doi:10.3758/s13414-017-1288-7) contains supplementary material, which is available to authorized users.

Nicholas E. DiQuattro

nickof4@gmail.com

Joy J. Geng

jgeng@ucdavis.edu

1 Department of Psychology, University of California, Davis, Davis 95616, CA, USA

2 Center for Mind \& Brain, UC Davis, 267 Cousteau Pl, Davis, CA 95618, USA
Keywords Attention · Selective, Eye Movements · Mechanisms, eye movements and visual attention

Although the perceptual details of the world are infinite, the capacity of the visual system is limited. These limitations require strategic deployment of cognitive resources to focus processing on only the most relevant information for current goals. Attention is the mechanism that achieves this by selecting relevant information over the irrelevant. However, attentional control is susceptible to errors that result in the occasional selection of irrelevant information. These errors result most commonly from the capture of attention by objects that are task irrelevant, but perceptually salient (Theeuwes, 1994, 2004) or objects that possess target-similar features (Folk, Remington, \& Johnston, 1992). Although both salient and target-similar stimuli capture attention, several studies have shown that salient distractors can be "rapidly rejected," as characterized by hyper-short dwell times or limited behavioral interference (but see Born \& Kerzel, 2009; Fukuda \& Vogel, 2009, 2011; Gaspelin, Leonard, \& Luck, 2015; Geng \& DiQuattro, 2010; Ludwig \& Gilchrist, 2002; Vatterott \& Vecera, 2012). Rapid rejection has been hypothesized to occur because of the strong mismatch between the distractor and target features, but it remains unclear what role target competition plays. In the present studies, we explore the necessity of feature-based competition for the rapid attenuation of attention to dissimilar distractors.

Eye movements have been used to track attentional deployment in a number of studies, and the fact that shifts of attention precede saccades is well established (Deubel \& Schneider, 1996; Hoffman \& Subramaniam, 1995; Kowler, Anderson, Dosher, \& Blaser, 1995). Eye-movement data are therefore frequently used as an overt metric of attentional capture. For example, Godijn and Theeuwes (2002) tracked eye 
movements while participants viewed an array of red circles arranged in an imaginary circle around a fixation cross. After $600 \mathrm{~ms}$, one of the circles changed to gray, identifying it as the target. On a subset of trials, an additional red circle appeared in a new location simultaneous with the target. Analysis of the first saccade on each trial showed that the onset distractor captured attention and the first saccade $28.5 \%$ of the time despite occurring in an entirely task-irrelevant location. Saccades on these trials had shorter latencies and dwell times (i.e., intersaccadic intervals) compared to target-bound saccades. This suggested the presence of a corrective mechanism following oculomotor capture by a salient distractor in a task-irrelevant location. Subsequent studies replicated these results and found that shorter dwell times occurred not only on distractors in task-irrelevant locations but also ones with features that were highly dissimilar from the target (Born, Kerzel, \& Theeuwes, 2011; Geng \& DiQuattro, 2010). This led to the suggestion that attention was rapidly disengaged from the distractor because of the strong mismatch between its properties and those of the target template (Geng, 2014; Theeuwes, 2010).

In contrast to the disengagement hypothesis, the simultaneous programming of two competing saccades has also been posited as an explanation for shorter dwell times on irrelevant objects (McPeek, Skavenski, \& Nakayama, 2000; Theeuwes, Kramer, Hahn, \& Irwin, 1998). Given that a typical saccade requires $150-250 \mathrm{~ms}$ to initiate (Findlay, 1997), short dwell times on the first saccade target could result from competing oculomotor plans that were initiated prior to the completion of the first saccade. Theeuwes, Kramer, Hahn, Irwin, and Zelinsky (1999) described an "independent horse-race" model in which distinct saccade plans to two objects race toward a threshold to determine which plan is executed first. In their model, capture by a dissimilar distractor occurs because its saccade plan reaches threshold first, but the dwell time is short because the second saccade is initiated prior to completion of the first saccade. Thus, the movement of attention away from the distractor is due to competition from a target alternative, not because processing of the first saccade target was actively suppressed because of the identification of task-irrelevant features.

Thus, despite clear evidence that oculomotor capture by a target-dissimilar distractor object can be rapidly rejected, the informational requirements of this mechanism remain unclear. One hypothesized source of information is from "distractor identity" where distractor suppression is rapid because of the mismatch between the object and an internal representation of the task-relevant target; another, nonmutually exclusive, source is the presence of a target (or target-similar) object at an alternative location, which causes concurrent saccade planning. The former is based on the recognition that the current saccade target is incorrect and the latter is based on the presence of a second saccade plan toward a more goal-relevant object. These two mechanisms have been hypothesized to co-occur in "step" tasks, where the target shifts locations before the first saccade (Camalier et al., 2007). A comparison of race models with and without the "stop" signal found that the data were best described by both a "stop" signal for the current saccade and an alternative "go" signal to the target in the new location (Camalier et al., 2007). This suggests that the ability to suppress distractor processing depends on competition that is sensitive to the feature similarity of the distractor to the target, as well as the presence of an object that better fits the target identity. The purpose of our studies was to better understand how the timing of competition from a target-like object influences the speed of distractor rejection.

\section{Experiment 1}

The goal of this experiment was to validate our current paradigm by replicating previous findings of short dwell times on target-dissimilar distractors accompanied by performance enhancements. Participants performed a visual search task in which the target appeared either with a similar or dissimilar distractor. We expected dwell time to be shorter on dissimilar compared to similar distractors and that this would correspond with shorter reaction time and higher accuracy.

\section{Method}

Participants Twenty-four UC Davis undergraduates (11 male, three left-handed, ages 19-27 years) participated for class credit. Each provided written informed consent in accordance with the local ethics clearance, as approved by the National Institutes of Health. All had normal or corrected-tonormal vision.

Procedure A central fixation dot (subtending $.25^{\circ}$ of visual angle; gray; $8.51 \mathrm{~cd} / \mathrm{m} 2$ ) was centrally presented on a black background $(.23 \mathrm{~cd} / \mathrm{m} 2)$ for the duration of the experiment. On each trial, a diamond and a square (each subtending $1^{\circ}$ of visual angle) appeared for $500 \mathrm{~ms}$ (see Fig. 1). The target was always the diamond-shaped object that appeared at one of six locations along an imaginary circle (radius of $5^{\circ}$ visual angle); the distractor was always a square that appeared at the antipode location. Each object contained a $t$ that was either upright or inverted. Participants were instructed to report the orientation of the $t$ within the target object by a right-handed key press. The $k$ key indicated an upright $t$ and the $m$ key an inverted $t$. The target color remained constant throughout the experiment (e.g., green), while the distractor was white (198 $\mathrm{cd} / \mathrm{m} 2,30.3 \%$ of trials), purple $(9.8 \mathrm{~cd} / \mathrm{m} 2,30.3 \%$ of trials $)$, or the same color as the target (e.g., green; $17.1 \mathrm{~cd} / \mathrm{m} 2 ; 30.3 \%$ of trials). The target color (green or purple) was counterbalanced between participants. After the search display, an intertrial 
A

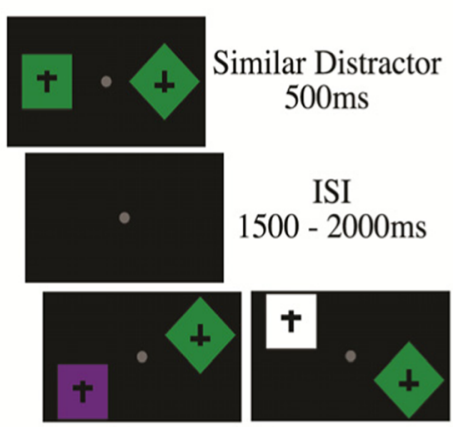

Dissimilar Distractors

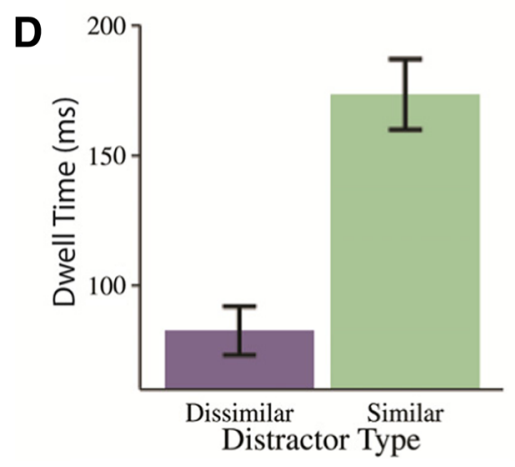

B

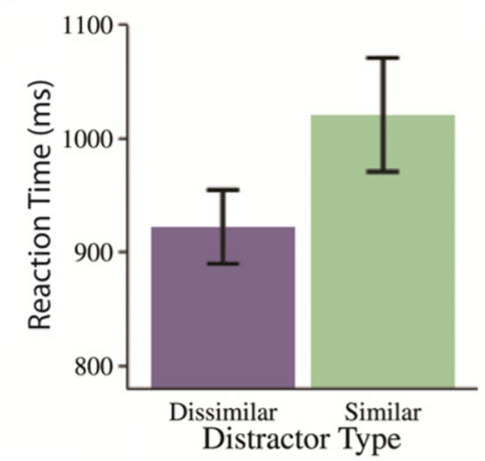

E

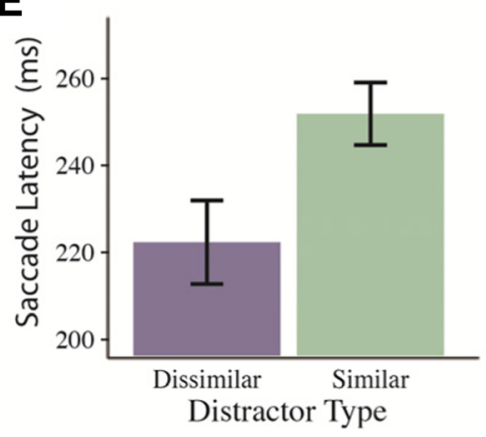

C

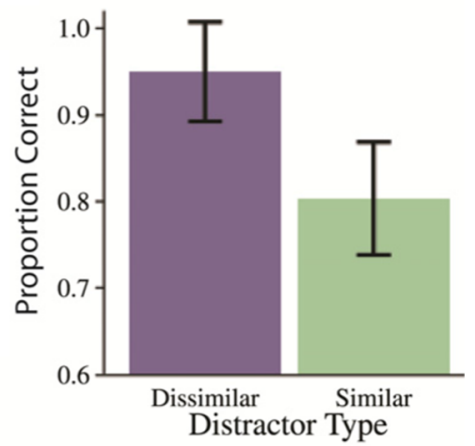

Fig. 1 A Experimental design for Experiment 1. Participants were instructed to indicate the orientation of the cross within the target object (green diamond). Distractors were always square shaped and could occur in a target similar or two dissimilar colors. "Catch" trials (not depicted) included two of the square stimuli and no target. The two dissimilar

interval of 1,500-2,000 ms occurred with only the fixation dot visible. On a subset of trials (9\%), no target appeared (i.e., the task display consisted of two distractors, both square shaped), in which case the participants were instructed to withhold a response. These "catch trials" consisted of two square nontargets. One configuration had a green (target-colored) square with a white or purple square; the other had one white and one purple square (i.e., two nontarget colors). There were a total of 534 trials.

Apparatus Participants were seated in a sound-attenuated room $70 \mathrm{~cm}$ away from a 24-in. Dell LCD monitor with a spatial resolution of $1,900 \times 1,200$ pixels and a refresh rate of $60 \mathrm{~Hz}$. Prior to the start of each trial, fixation of the centrally presented gray dot was enforced for $100 \mathrm{~ms}$ via an Eyelink 1000 (SR Research, ON) desk-mounted system sampling at $500 \mathrm{~Hz}$. Eye movements were also recorded for the duration of the task display.

Analysis There were no significant differences in manual RT, manual accuracy, or dwell time between trials with the two nontarget colors (e.g., white and purple), all $t \mathrm{~s}(22)<.73, p \mathrm{~s}>$ .47. We therefore combined data from those trials (one subject was not included because of lack of any saccades to one distractor). This resulted in two distractor conditions, the distractors were collapsed in all analyses (see Method section). B-E Manual reaction time, proportion correct, distractor dwell time, and saccade latency from trials where the first saccade was directed toward a distractor. Error bars represent $95 \%$ confidence intervals (Color figure online)

"dissimilar" condition with target-dissimilar colored distractors and the "similar" condition with target-colored distractors. Means and confidence intervals for conditions not discussed are available in the supplementary materials.

A saccade was classified as such if the velocity exceeded $30 \%$ second. The saccade was determined to have been made to an object if it landed within $2.5^{\circ}$ from the center of an object. Dwell times were calculated as the total time during which an object was continuously fixated. The duration of multiple small fixations within the interest area were summed if the eyes never left the interest area. The dwell time therefore reflects the total duration that an object was examined continuously. Saccade latency was calculated as the duration from the onset of the trial until the eyes left the fixation interest area, defined by a box with center-to-edge distance of $2.5^{\circ}$ visual angle. Because the eyes were only tracked during the 500-ms search display, we could not reliably analyze second saccades. Although subjects must have looked at targets after distractors to respond correctly, we were not able to analyze the eye metrics associated with the second saccade (e.g., dwell time).

\section{Results}

Overall manual response accuracy was high $(M=91.52 \%, S D$ $=6.7 \%$ ), indicating that participants understood and were able 
to perform the task. Participants had shorter manual RT, $t(23)$ $=-4.22, p<.001, d=.86$, and higher accuracy, $t(23)=5.14, p$ $<.001, d=1.05$, on trials with dissimilar distractors compared to similar distractors (see Fig. 1). Consistent with the manual RT and accuracy data, there were fewer distractor-first saccades in the dissimilar distractor compared to a similar distractor conditions, $t(23)=-12.9, p<.001, d=2.64$, dissimilar: $M=11.5 \%, 95 \%$ CI $[8.15,14.86]$; similar: $M=39.3 \%$, $95 \%$ CI [35.31, 43.21]. Subsequent analyses of data include only trials in which the first saccade was directed to a distractor and the manual response was correct.

The dwell time was significantly shorter on dissimilar compared to similar distractors, $t(23)=16.9, p<.001, d=3.44$, replicating previous findings. These results replicate previous results and demonstrate that dissimilar distractors are rejected more rapidly than similar ones and have an overall lower cost on performance (DiQuattro \& Geng, 2011; Geng \& DiQuattro, 2010; Mazaheri, DiQuattro, Bengson, \& Geng, 2011). Similarly, saccades to dissimilar distractors had shorter latencies than similar distractors, $t(23)=7.05, p<.001$ (see Fig. 1). The pattern of distractor dwell times and latencies are consistent with race models of oculomotor control in which saccades to dissimilar distractors only occur when evidence in favor of the distractor accumulates rapidly (Camalier et al., 2007; Geng \& DiQuattro, 2010; Godijn \& Theeuwes, 2002). However, because competition from the target is high, dwell times on the dissimilar distractor were short, indicating the rapid shift of prioritization to a stimulus that matched top-down goals.

In addition to the target-present trials, we analyzed the catch trials in which two dissimilar distractors were presented in the display. Interestingly, the dwell times on dissimilar distractors in these catch trials were comparable to those of similar distractors from the target-present trials, $M=173.56 \mathrm{~ms}, 95 \%$ CI [160.05, 187.07], and significantly longer than dwell times of dissimilar distractors on target-present trials, $t(23)=19.72, p<.001, d=4.03$. Shortened dwell times on dissimilar distractors only occurred when a target was present in the display, suggesting that the time to reject a distractor depended on the presence of competition from a more target-like object. The latency data showed a similar pattern such that saccade latencies to a distractor on catch trials, $M=264.24 \mathrm{~ms}, 95 \% \mathrm{CI}$ [254.54, 273.95], were similar to the latency to similar distractors when a target was present, but longer than latencies to dissimilar distractors presented with a target, $t(23)>7.05, p<.001$. However, the cause of longer dwell times and latencies were unclear from these data. For example, instead of continuing to process the distractor, they may have reflected uncertainty about where to go next given the absence of a target-like object. We sought to better understand the influence of target presence on distractor dwell times in the next two experiments.

\section{Discussion}

The goal of Experiment 1 was to replicate previous findings that target-dissimilar distractors are rapidly rejected. As expected, we found shorter latencies and dwell times on target-dissimilar distractors compared to target-similar distractors. There were also shorter RTs and higher accuracy on dissimilar distractor trials. This replicates previous findings in which target-dissimilar distractors had short dwell times indicating rapid rejection. Analysis of catch trials with two dissimilar distractors showed longer dwell times compared to the same object when paired with a target. This result suggests that the shorter dwell times observed on target-present trials were due to the presence of a target-like alternative. However, the catch trials results could also be explained by a general cessation of search after the first saccade or an inhibition of other saccades to the second dissimilar object.

\section{Experiment 2}

In Experiment 2, we tested the consequences of delaying the target onset on distractor rejection. In the current experiment, we added a stimulus onset asynchrony ( $\mathrm{SOA}$ ) between the onset of distractor and target $(0 \mathrm{~ms}, 33 \mathrm{~ms}, 68 \mathrm{~ms}$, and 100 $\mathrm{ms})$. The opposite conditions in which the target appeared first were included to reduce temporal expectations, but were not conditions of primary interest. If the speed of rapid rejection relies on the initiation of a competing oculomotor plan to the target, then delaying the onset of the target should reduce the speed of distractor rejection commensurate with the length of the delay. Such a result would be consistent with race models in which two oculomotor plans are executed in parallel. Alternatively, if rapid rejection relies in part on discrimination of the distractor's identity (e.g., creating a "stop" signal), then the dwell time on distractors may be shorter than expected given the delay in target onset (Camalier et al., 2007).

\section{Method}

Participants Twenty-four UC Davis undergraduates (nine male, all right-handed, ages 18-28 years) participated for class credit. Each provided written informed consent in accordance with the local ethics clearance, as approved by the National Institutes of Health. All had normal or corrected-to-normal vision.

Design The experimental design was identical to Experiment 1, except that the target onset was delayed relative to distractor onset by a stimulus onset asynchrony (SOA) of $0 \mathrm{~ms}$ (i.e. no delay), $33 \mathrm{~ms}, 68 \mathrm{~ms}$, or $100 \mathrm{~ms}$. To avoid expectancies about the identity of the first onset, the distractor-first trials were balanced by the reverse pattern of target-first onsets (i.e., 
distractor onset was delayed relative to the target onset by the same intervals). In contrast to Experiment 1, there were no target-absent "catch" trials. Results from the target-first conditions are not of interest for the question of distractor rejection because they almost always resulted in target-first saccades; the results are therefore reported in the supplementary materials. There were 36 trials in each distractor type by onset delay condition, for a total of 756 trials.

Analysis The purpose of this study was to understand performance related to distractor processing. For this reason, we report only the data from trials in which the distractor appeared simultaneous with, or before, the target and on which the first saccade went to the distractor. Trials in which the target appeared first resulted in few to no saccades to the distractor and therefore are only reported in the supplemental materials. The criterion for saccades, dwell times, and latencies were identical to those in Experiment 1.

The number of trials with first saccades to the distractor increased with SOA for both dissimilar and similar distractor trials, means for each SOA in the dissimilar condition: no delay = $6.00(S D=3.12), 33 \mathrm{~ms}=14.46(S D=9.10), 68 \mathrm{~ms}=26.79(S D$ $=10.97), 100 \mathrm{~ms}=33.08(S D=13.06)$; means for each SOA in the similar condition: no delay $=8.79(S D=4.38), 33 \mathrm{~ms}$ $=13.00$ ( $S D=7.06), 68 \mathrm{~ms}=16.38(S D=6.50), 100 \mathrm{~ms}=$ $15.92(S D=7.64)$. It was expected that there would be relatively fewer trials in the no-delay condition given the low likelihood of the dissimilar distractor winning the competition for the first saccade, and therefore those data are never interpreted in isolation of the data form other SOA conditions. Analysis of trial numbers as a proportion of the total trials is reported in the Results section as a function of SOA and distractor type.

\section{Results}

For distractor-first saccades, manual RT was overall shorter for dissimilar distractors $F(1,23)=41.34, p$ $<.001, \eta^{2}=.64$, compared to similar distractors, but there was no effect of SOA, $F(3,69)=1.05, p=.38$, nor an interaction $F(3,69)=0.62, p=.6$. A similar pattern was found in manual response accuracy with dissimilar distractor trials showing significantly higher accuracy, $F(1,23)=41.98, p<.001, \eta^{2}=.65$, but no effect of SOA, $F(3,69)=0.75, p=.53$, nor an interaction, $F(3$, $69)=1.28, p=.3$. Together, these results replicate those of Experiment 1, showing improved behavioral performance when the first saccade is directed towards a dissimilar distractor compared to a similar one (see Fig. 2), but this main effect was not modulated by the distractor-to-target SOA (recall that we report here only trials in which the distractor appeared simultaneous with, or before, the target; see Method section). However, manual RT may be insensitive to SOA given that RT (approximately 900-1,100 ms) is coarse compared to the SOA manipulation (100 ms or less). We therefore turn next to the eye data.

Proportion of first saccades to the distractor was entered into a $2 \times 4$ ANOVA, which found significant main effects of distractor type, $F(1,23)=148.1, p<.001, \eta^{2}=.87$, and SOA, $F(3,69)=298.8, p<.001, \eta^{2}=.93$, but not an interaction effect, $F(3,69)=.1, p=.4$. The longer the target was delayed, the more likely the first saccade was directed towards the distractor. However, similar distractors were overall more likely to capture the first saccade than dissimilar ones, suggesting that competition was affected by both temporal precedence and stimulus identity.

The rate of first saccades to distractors was high, especially for longer distractor-to-target SOAs (see Fig. 2). We therefore next examined how the timing of target competition affected processing of distractors that won the competition for the first saccade. Dwell time was entered into a 2 distractor type (dissimilar, similar $) \times 4 \operatorname{SOA}(0,33,68,100 \mathrm{~ms})$ ANOVA, which found a significant main effect of distractor type, $F(1,23)=$ $403.7, p<.001, \eta^{2}=.95, \mathrm{SOA}, F(3,69)=25.52, p<.001, \eta^{2}$ $=.53$, and an interaction, $F(3,69)=3.32, p=.02, \eta^{2}=.13$. The main effect indicated that dwell time was shorter overall for dissimilar distractors, but the interaction showed a greater increase in dwell time on dissimilar distractors as the distractor-to-target SOA increased.

Saccade latencies were calculated beginning from distractor onset (before or simultaneous with the target) until the first saccade was executed. Using the same ANOVA as for dwell time, significant main effects were found for SOA, $F(3,69)=6.89, p<.001, \eta^{2}=.23$, and distractor type, $F(1,23)=67.36, p<.001, \eta^{2}=.75$. The interaction was also significant, $F(3,69)=4.17, p<.001$, $\eta^{2}=.15$ (see Fig. 2). Because the pattern of latencies was different than the dwell times, we also analyzed the combined latency + dwell time data to understand the total processing time using the same 2 distractor type $\times 4$ SOA ANOVA. There were significant main effects of distractor type, $F(1,23)=705.4, p<.001, \eta^{2}=.9$, and SOA, $F(3,69)=11.54, p<.001, \eta^{2}=.33$, and a significant interaction, $F(3,69)=8.0, p<.001, \eta^{2}=.26$. The interaction pattern was identical to that of dwell times alone (see Fig. 2): Latency + dwell times increased with the target delay more so in the dissimilar distractor condition compared to the similar distractor condition.

Together, these data show that the total processing time on dissimilar distractors increased as the delay in the appearance of target competition increased. This suggests that the appearance of the target initiated a second, corrective, saccade plan that cut off processing of the dissimilar distractor. The appearance of the target had less of an impact on processing of similar distractors, likely due to similar distractors being mistaken for possible targets. 
A

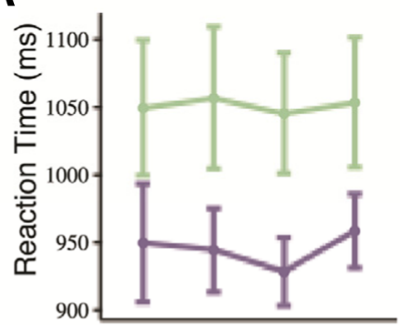

B

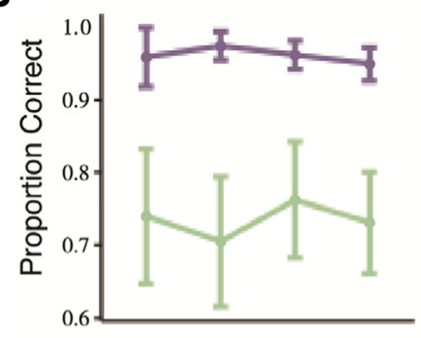

C

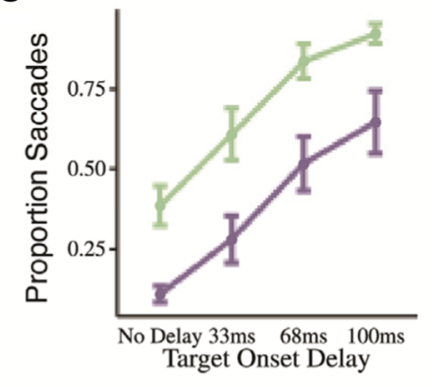

$\mathbf{F}$

D

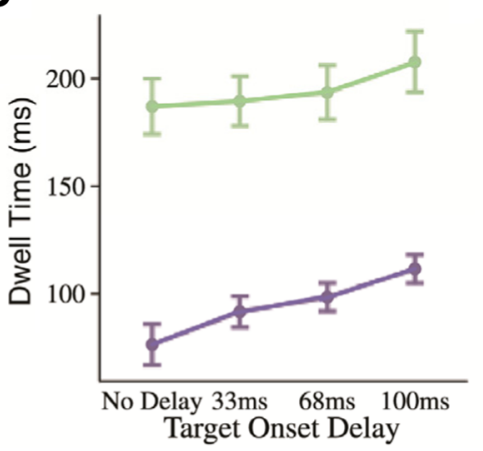

$\mathbf{E}$

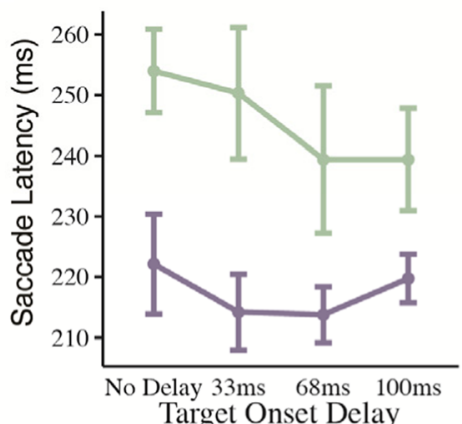

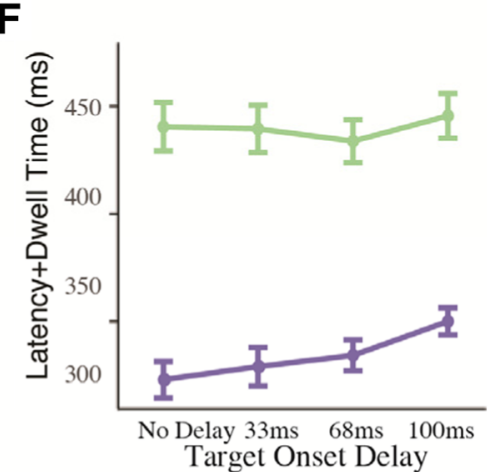

Fig. 2 A-F Manual reaction time, proportion correct, proportion of distractor-first saccades, fixation dwell time, saccade latency, and combined latency + dwell time in Experiment 3. Data in $\mathbf{a}-\mathbf{b}$ and $\mathbf{e}-\mathbf{f}$ are from trials with first saccades directed toward distractor. Error bars

\section{Discussion}

The goal of Experiment 2 was to test if the shorter processing time on dissimilar distractors found in Experiment 1 depended on the presence of a target in an alternative location during oculomotor planning. To test this question, we used a parametric delay in the onset of the target. The results showed a monotonic increase in dwell times on dissimilar distractors with longer delays in target onset, but there was no effect on similar distractors. This finding suggests that the speed to reject a distractor relies on the presence of competition from a more target-like alternative. However, the increase in processing time on dissimilar distractors was less than the actual duration of the delay in target appearance. This suggests dwell times were affected by not only the initiation of an oculomotor plan to the target with a fixed time to execute but also the presence of a "stop" signal to the ongoing saccade plan to the dissimilar distractor (Camalier et al., 2007). Dwell times were also shorter for dissimilar distractors overall, suggesting that the ease of distinguishing the distractor from the target contributed to the ability to generate the "stop" signal. In contrast, the similar distractor could only be distinguished from the target based on shape, which in this study appears to be less salient than color (Theeuwes, 1991, 1992). are $95 \%$ confidence intervals. Both dwell time and latency + dwell time showed that delay in target onset had a larger effect on dissimilar distractors than on similar distractors (Color figure online)

\section{Experiment 3}

In Experiment 3, we tested if the pattern of short distractor fixations would be eliminated if the presence of a target were delayed until after the first saccade was initiated using a saccade-contingent display. Saccadic suppression prevents visual processing during a saccade (Bridgeman, Hendry, \& Stark, 1975; Matin, 1974) and therefore affords us the ability to change the stimulus array without creating a local onset alerting subjects to the change. This allowed us to examine the degree to which dwell times on dissimilar distractors are influenced by competition from a target object during initial oculomotor planning compared to competition that occurs after the first saccade. Because the use of a saccade-contingent display increased the complexity of the experimental design, only dissimilar distractors were included (i.e., conditions with similar distractors in Experiments 1 and 2 were excluded from this study).

\section{Method}

Participants Twenty-four UC Davis undergraduates (seven male, all right-handed, ages 18-26 years) participated for class credit. Each provided written informed consent in accordance with the local ethics clearance, as 
approved by the National Institutes of Health. All had normal or corrected-to-normal vision.

Design The experimental design was identical to Experiment 1, with the following differences: Both the eccentricity and size of the target/distractor items were increased (displayed at $10^{\circ}$ visual angle from screen center and subtending $2^{\circ}$ visual angle) to allow for saccade contingent display switching. The search display remained on until a response was made. On target-absent trials, participants were instructed to press the space bar. There were four possible initial displays: a single distractor (DistAlone), a single target (TargAlone), two distractors (DistDist), or a distractor and a target (DistTarg). On $50 \%$ of trials, the search array stayed unchanged and on the other $50 \%$, the display changed during the first saccade. The possible change trial types are illustrated in Fig. 3: on DistAlone-to-DistTarg trials, the initial screen only had a single distractor, but a target appeared in the antipode location after the first saccade was executed; on TargAlone-to-DistTarg trials, a single target was present initially, but a distractor appeared in the antipode location once the first saccade was initiated; on DistDist-to-DistTarg trials, the distractor in the location opposite to the saccade target changed into a target during the saccade; and finally, on DistTarg-to-DistDist trials, the target changed into a distractor, but only if the saccade was directed toward the distractor.

A full factorial experimental design was used to eliminate expectations regarding the relationship between the initial display and final outcomes. However, the conditions of greatest interest only included the one with target competition before the first saccade (i.e., DistTarg-to-DistDist) and those with target competition only after the first saccade (i.e., DistDist-to-DistTarg and DistAlone-to-DistTarg). The timing of the target in these conditions resulted in conditions with target competition only in the pre-saccade period or only in the post-saccade period. Each initial display condition had 150

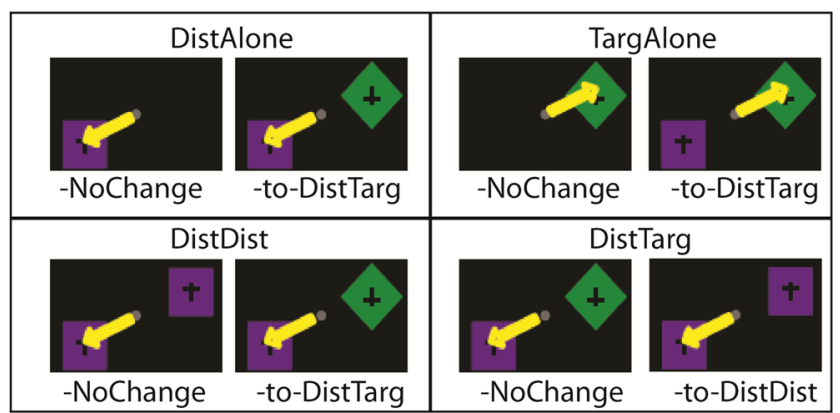

Fig. 3 Experimental design for Experiment 3. Yellow arrow indicates direction of first saccade. Top label describes contents of initial display; bottom labels describe postsaccade display. Participants were instructed to report the orientation of the cross within the target (e.g., green diamond) or indicate target absence with a separate button press (Color figure online) trials, for a total of 600 trials. Participants also completed a practice block consisting of 15 trials.

Analysis The data analyses were restricted to trials in which the first saccade was directed to a distractor. The critical conditions were the DistAlone-to-DistTarg condition, which had an average of $54.27(S D=11.36)$ trials; the DistDist-to-DistTarg condition, which had 48.07 ( $S D=$ 17.87) trials; and the DistTarg-to-DistDist condition which only had an average of $4.13(S D=4.00)$. This was because some subjects did not make eye movements to the distractor when a target was initially present. We therefore excluded these subjects from subsequent analyses in which insufficient trials with distractor saccades occurred. This resulted in fewer subjects included in analyses with the DistTarg-to-DistDist condition $(n=15)$. Results from target-bound saccades are reported in the supplementary materials.

Apparatus To update search displays during saccades, stimuli were displayed on an Asus MG279Q 27-in. monitor with a refresh rate of $144 \mathrm{~Hz}$ and a resolution of 2,560 $\times 1,440$. Eyetracking equipment and settings remained identical to Experiments 1 and 2.

\section{Results}

Overall manual response accuracy was high $(M=95.57 \%, S D$ $=3.34 \%$ ), indicating that participants understood the task. The primary question of interest was whether the timing of the target's presence (before or after the first saccade) would affect distractor processing, when the first saccade was directed to the distractor. To address this, we compared the three conditions of interest in which the target was either paired with a distractor in the initial display or only after the first saccade (i.e., DistTarg-to-DistDist vs. DistDist-to-DistTarg and DistAlone-to-DistTarg) using a one-way ANOVA. Recall that analyses were only conducted on trials where the first saccade was directed to the distractor. Manual accuracy was significantly different, $F(2,28)=15.52, p<.001, \eta^{2}=.53$; but manual RT was not, $F(2,28)=.79, p=.47$. Pairwise $t$ tests of the accuracy data showed higher accuracy in the DistTarg-to-DistDist condition compared to the two conditions when the target only appeared after the first saccade, both $t \mathrm{~s}(14)>4.7, p \mathrm{~s}<.001, d \mathrm{~s}=1.80$ (see Fig. 4). There was also a marginal difference between the two conditions with the postsaccade target, $t(14)=2.1, p=.055, d=.60$, suggesting that accuracy was higher when there was only one distractor initially. Recall that the first saccade was directed to the distractor on all of these trials; therefore, the results indicate that performance was better when the target was present in the initial display, even if the distractor won the competition for the first saccade. 

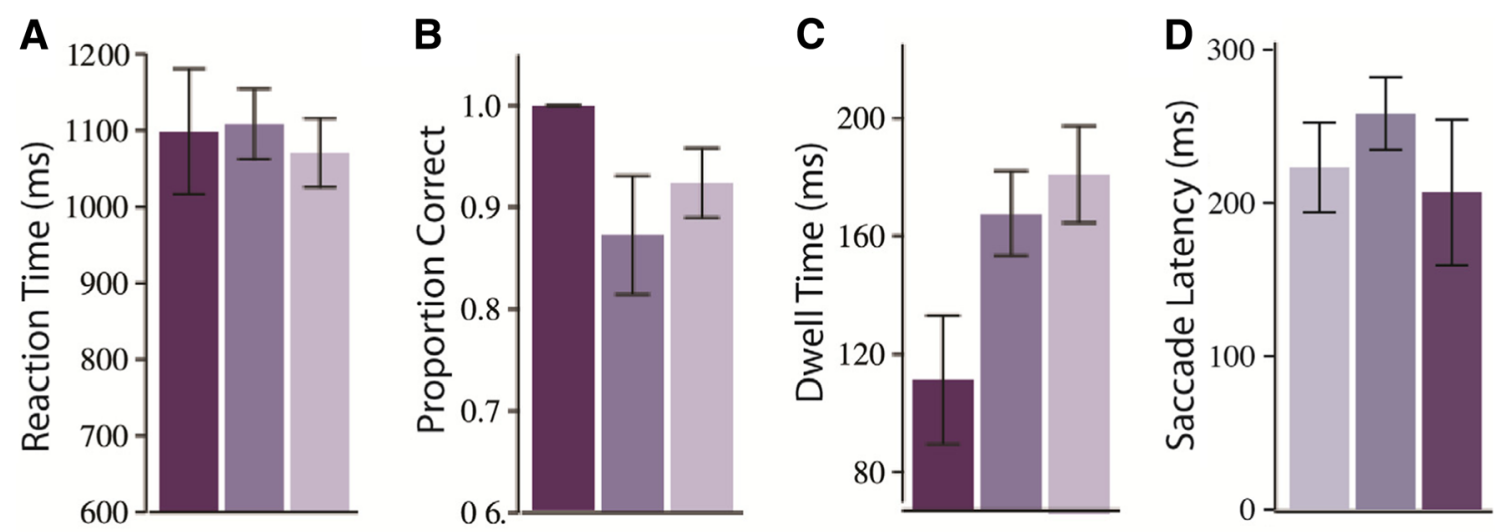

DistTarg-to-DistDist

DistDist-to-DistTarg

DistAlone-to-DistTarg

Fig. 4 A-D Manual reaction time, accuracy, fixation dwell time, and saccade latency data from Experiment 3 in critical conditions of comparison when the target was initially present in the display versus when the target only appeared after the first saccade. All error bars are $95 \%$ confidence intervals

Consistent with the manual accuracy data, distractor dwell times were shorter when the target was present in the initial display compared to both conditions when the target appeared only after the first saccade, $F(2,28)=29$, $p<.001, \eta^{2}=.67$, both $t \mathrm{~s}(14)>4.8, p \mathrm{~s}<.001, d \mathrm{~s}>1.88$. Dwell times were also shorter in the DistDist-to-DistTarg conditions compared to the DistAlone-to-DistTarg condition, $t(14)=3.4, p<.005, d=.47$, suggesting that initial competition from a second distractor resulted in shorter dwell times. Saccade latencies showed a similar pattern, $F(2,28)=4.72, p=.02, \eta^{2}=0.25$, but now the difference was only significant between the DistTarg-to-DistDist and DistDist-to-DistTarg conditions, $t(14)=2.5, p<.05, d=$ .61 (see Fig. 4). Interestingly, also similar to dwell time, there was a significant difference between the two conditions with only postsaccade targets, $t(14)=9.1, p<.001$, $d=.88$, suggesting again that the presence of distractor competition in the presaccade display also had an effect on performance, but not as dramatically as when a target was present initially. Together, these results are consistent with those from the previous two experiments in showing that the presence of target competition in the initial display is necessary to produce short distractor dwell times; they go farther by showing that the continued presence of the target after the saccade is unnecessary.

The previous data show that accuracy was higher and distractor dwell times shorter when a plan for a second, corrective saccade was queued before the execution of the first saccade. This was true even though the target disappeared postsaccade. However, the condition in which the target was initially present contained very few trials because subjects rarely made first saccades to distractors when a target was present. Therefore, we sought convergent evidence for the conclusion that target competition needs to be present prior to the first saccade for short dwell times to occur, by testing whether the appearance of a target postsaccade would be sufficient to produce shortened distractor dwell times. To do this, we compared the three conditions that contained only distractors in the initial display (i.e., DistAlone-to-DistTarg, DistDist-to-DistTarg, and DistDist-NoChange). All of these conditions contained an average of more than 40 trials each (see Method section).

If the postsaccade target affected distractor processing, then dwell times should be shorter when a target appeared postsaccade compared to when it did not. However, if the postsaccade display has no effect on distractor processing, as hypothesized, then there should be no difference in dwell times based on the appearance of the target postsaccade. Consistent with the latter hypothesis, there were no significant differences between the three conditions, $F(2,46)=0.68, p=$ .512; DistAlone-to-DistTarg $=188.43 \mathrm{~ms}, 95 \%$ CI [176.63, 200.22], DistDist-to-DistTarg $=191.43 \mathrm{~ms}, 95 \%$ CI [170.02, 212.85, and DistDist-NoChange $=196.23 \mathrm{~ms}, 95 \% \mathrm{CI}$ [180.33, 212.12]. The appearance of the target after the saccade did not affect dwell times. This extends the results from "catch" trials in Experiment 1 by showing that the long dwell times on distractors were not due to an absence of "somewhere to go" but reflect the lack of competition during the previous fixation.

Finally, we compared the current results against those from Experiment 2 to see if dwell times were longer when the target only appeared postsaccade than when the target onset was delayed for $100 \mathrm{~ms}$ (i.e., the longest delay used). There were two conditions in this experiment where the target appeared after the first saccade (i.e., DistAlone-to-DistTarg and DistDist-to-DistTarg), and each was compared against the $100 \mathrm{~ms}$ delay condition in Experiment 2. Both comparisons were significant, DistDist-to-DistTarg, $t(37)=8.42, p<.001$, $d=1.37$; DistAlone-to-DistTarg, $t(37)=9.53, p<.001, d=$ 1.55. Distractor dwell times when the target was delayed until after the first saccade were even longer than when the target was delayed for $100 \mathrm{~ms}$. 


\section{Discussion}

The goal of Experiment 3 was to test if and when the presence of a competing target is required for rapid rejection of a dissimilar distractor. Our results indicated that the information in the initial display configuration determined the dwell time on dissimilar distractors in all conditions: If a target was initially present, dwell times were short, but if the target only appeared after the first saccade was initiated, dwell times were longer. This suggests that the identity of the distractor alone was not sufficient to produce the short dwell times seen during rapid distractor rejection. The presence of target competition during the initial fixation determined the dwell time and speed of attentional disengagement. These results indicate that in order for rapid rejection to occur for a dissimilar distractor, a competing target must not only be present but must appear during saccade planning.

\section{General discussion}

Previous findings have demonstrated the ability of the attentional system to rapidly reject distractors that were clearly nontargets (Born et al., 2011; DiQuattro \& Geng, 2011; Fukuda \& Vogel, 2009, 2011; Geng, 2014; Geng \& DiQuattro, 2010; Godijn \& Theeuwes, 2002; Mazaheri et al., 2011; Theeuwes, 2010). However, it was unclear in those studies whether the short dwell times on distractors were due to the identity of the distractor being strongly mismatched to task-relevant features, or due to the presence of strong competition from another object with target-like features. Here, we extended previous results by measuring the dependence of rapid distractor disengagement (i.e., shorter dwell times) on distractors as a function of a competing target at an alternative location. We found an interaction between distractor identity and SOA on distractor processing: delaying the onset of the target resulted in a monotonic increase in dwell times on dissimilar distractors, but had limited effect on similar distractors (Experiment 2); furthermore, if the appearance of the target was delayed until after the first saccade, the dwell time was no longer different than when the target was completely absent (Experiment 3). This suggests that the presence of competition from a target (Desimone and Duncan, 1995), prior to the initiation of the first saccade, was necessary for the rapid disengagement of attention from a dissimilar distractor.

Evidence that the alternative object had to be target-like was found on trials in which the two initial objects were both dissimilar distractors (Experiment 3). On these trials, dwell times on the distractor were longer than when a target was initially present, even when the other distractor had changed into a target during the saccade. The fact that a target-like alternative object had to be present before the first saccade was initiated suggests that attentional competition proceeds in parallel with oculomotor execution: Even when a saccade was being prepared to a dissimilar distractor, the appearance of a target generated a secondary, competing oculomotor plan reduced dwell times on the distractor. This result is consistent with findings by Born et al. (2011) in which they found that the time to disengage from a distractor does not depend on its initial likelihood of capturing attention but depends on information about alternative target-like objects that are present before the saccade to the distractor is initiated.

These results combine work demonstrating that attention precedes saccades (Deubel \& Schneider, 1996; Kowler et al., 1995) with race models of oculomotor control. Race models describe why short intersaccadic fixation intervals occur when a second oculomotor plan is initiated before the first is finished (Boucher, Palmeri, Logan, \& Schall, 2007; Camalier et al., 2007; Godijn \& Theeuwes, 2002; McPeek et al., 2000). Standard models involve two oculomotor plans that evolve in parallel, but those models cannot fully describe our data because there was always a large difference in distractor dwell times based on the similarity of its color to the target. Dissimilar distractors were less likely to capture attention and the first saccade, and latencies and dwell times were uniformly shorter on these distractors than target-similar distractors. Furthermore, while distractor dwell times on the dissimilar distractors in Experiment 2 increased with the delay in target onset in Experiment 2 (unlike similar distractors), the increase in dwell times were substantially less than the delay. This suggests that attentional mechanisms sensitive to the mismatch of distractor features also contribute to dwell times.

The race architecture of the Camalier et al. (2007) model can explain our pattern of data because in addition to a race between two "go" signals, it also incorporates a "stop" signal on the first saccade when it is known to be an error. Although their studies were conducted with "step" tasks in which the location of the target shifted after a period of time, their findings suggest that competition for oculomotor control is influenced by target competition as well as inhibition of a saccade recognized to be incorrect. In our study, this "error" signal appeared to only be generated for dissimilar distractors. This can explain why the increase in dwell time was less than the target onset delay, and also why dwell times on similar distractors were minimally affected by the presence of the actual target: When the distractor could be mistaken for the target until late into processing, the second oculomotor plan was delayed. However, when the identity of the distractor could easily preclude it as a target, processing was inhibited in conjunction with development of an oculomotor plan to a better alternative. Interestingly, this was all determined by the contents of the visual display prior to the first saccade.

Together, these results suggest that the suppression of distractor processing is a consequence of rapid shifts of attention that select and evaluate incoming information continuously, even while a first saccade plan is still being formulated 
for execution. The information available during the "first glance" included the relevance of the saccade target as well as the presence of target-like alternatives. These bits of information determined the speed of distractor disengagement. Interestingly, the identity of the objects present just after the saccade landed did not have an effect on distractor dwell times. This suggests the presence of a delay in incorporating new information immediately after a saccade has occurred, even while information accumulation appears to continue until the next saccade is executed. The current studies extend previous findings by demonstrating the effect of the target onset on distractor processing. However, work is still needed to understand exactly how information about the identity of the distractor and target-like alternatives is combined to drive attentional priority and oculomotor behaviors.

Author note Nicholas E. DiQuattro and Joy J. Geng, Department of Psychology, Center for Mind \& Brain, University of California, Davis.

This research was supported by National Science Foundation Grant BCS-1230377-0.

Special thanks to Teresa Nguyen for her help in data collection.

\section{References}

Born, S., \& Kerzel, D. (2009). Congruency effects in the remote distractor paradigm: Evidence for top-down modulation. Journal of Vision 9(9): 3-3.

Born, S., Kerzel, D., \& Theeuwes, J. (2011). Evidence for a dissociation between the control of oculomotor capture and disengagement. Experimental Brain Research, 208(4), 621-631. doi:10.1007/ s00221-010-2510-1

Boucher, L., Palmeri, T. J., Logan, G. D., \& Schall, J. D. (2007). Inhibitory control in mind and brain: An interactive race model of countermanding saccades. Psychological Review, 114(2), 376-397. doi:10.1037/0033-295X.114.2.376

Bridgeman, B., Hendry, D., \& Stark, L. (1975). Failure to detect displacement of the visual world during saccadic eye movements. Vision Research, 15(6), 719-722.

Camalier, C. R., Gotler, A., Murthy, A., Thompson, K. G., Logan, G. D., Palmeri, T. J., \& Schall, J. D. (2007). Dynamics of saccade target selection: Race model analysis of double step and search step saccade production in human and macaque. Vision Research, 47(16), 2187-2211. doi:10.1016/j.visres.2007.04.021

Desimone, R., \& Duncan, J. (1995). Neural mechanisms of selective visual attention. Annual Review of Neuroscience, 18(1), 193-222. doi:10.1146/annurev.ne.18.030195.001205

Deubel, H., \& Schneider, W. X. (1996). Saccade target selection and object recognition: Evidence for a common attentional mechanism. Vision Research, 36(12), 1827-1837. doi:10.1016/0042-6989(95) 00294-4

DiQuattro, N. E., \& Geng, J. J. (2011). Contextual knowledge configures attentional control networks. Journal of Neuroscience, 31(49), 18026-18035. doi:10.1523/JNEUROSCI.4040-11.2011

Findlay, J. M. (1997). Saccade target selection during visual search. Vision Research, 37(5), 617-631. doi:10.1016/S0042-6989(96) 00218-0

Folk, C. L., Remington, R. W., \& Johnston, J. C. (1992). Involuntary covert orienting is contingent on attentional control settings. Journal of
Experimental Psychology: Human Perception and Performance, 18(4), 1030-1044. doi:10.1037//0096-1523.18.4.1030

Fukuda, K., \& Vogel, E. K. (2009). Human variation in overriding attentional capture. Journal of Neuroscience, 29(27), 8726-8733. doi:10. 1523/JNEUROSCI.2145-09.2009

Fukuda, K., \& Vogel, E. K. (2011). Individual differences in recovery time from attentional capture. Psychological Science, 22(3), 361368. doi:10.1177/0956797611398493

Gaspelin, N., Leonard, C. J., \& Luck, S. J. (2015). Direct evidence for active suppression of salient-but-irrelevant sensory inputs. Psychological Science, 26(11), 1740-1750. doi:10.1177/ 0956797615597913

Geng, J. J. (2014). Attentional mechanisms of distractor suppression. Current Directions in Psychological Science, 23(2), 147-153. doi: 10.1177/0963721414525780

Geng, J. J., \& DiQuattro, N. E. (2010). Attentional capture by a perceptually salient non-target facilitates target processing through inhibition and rapid rejection. Journal of Vision, 10(6), 1-12. doi:10.1167/ 10.6 .5

Godijn, R., \& Theeuwes, J. (2002). Programming of endogenous and exogenous saccades: Evidence for a competitive integration model. Journal of Experimental Psychology: Human Perception and Performance, 28(5), 1039-1054. doi:10.1037//0096-1523.28.5.1039

Hoffman, J. E., \& Subramaniam, B. (1995). The role of visual attention in saccadic eye movements. Perception \& Psychophysics, 57(6), 787795.

Kowler, E., Anderson, E., Dosher, B., \& Blaser, E. (1995). The role of attention in the programming of saccades. Vision Research, 35(13), 1897-1916.

Ludwig, C. J. H., \& Gilchrist, I. D. (2002). Stimulus-driven and goaldriven control over visual selection. Journal of Experimental Psychology: Human Perception and Performance, 28(4), 902-912.

Matin, E. (1974). Saccadic suppression: A review and an analysis. Psychological Bulletin, 81(12), 899-917.

Mazaheri, A., DiQuattro, N. E., Bengson, J., \& Geng, J. J. (2011). Prestimulus activity predicts the winner of top-down vs. bottom-up attentional selection. PLOS ONE, 6(2), e16243. doi:10.1371/ journal.pone. 0016243

McPeek, R. M., Skavenski, A. A., \& Nakayama, K. (2000). Concurrent processing of saccades in visual search. Vision Research, 40(18), 2499-2516. doi:10.1016/S0042-6989(00)00102-4

Theeuwes, J. (1991). Cross-dimensional perceptual selectivity. Perception \& Psychophysics, 50(2), 184-193.

Theeuwes, J. (1992). Perceptual selectivity for color and form. Perception \& Psychophysics, 51(6), 599-606.

Theeuwes, J. (1994). Stimulus-driven capture and attentional set: Selective search for color and visual abrupt onsets. Journal of Experimental Psychology: Human Perception and Performance, 20(4), 799-806. doi:10.1037//0096-1523.20.4.799

Theeuwes, J. (2004). Top-down search strategies cannot override attentional capture. Psychonomic Bulletin \& Review, 11(1), 65-70. doi: 10.3758/BF03206462

Theeuwes, J. (2010). Top-down and bottom-up control of visual selection. Acta Psychologica, 135(2), 77-99. doi:10.1016/j.actpsy.2010. 02.006

Theeuwes, J., Kramer, A. F., Hahn, S., \& Irwin, D. E. (1998). Our eyes do not always go where we want them to go: Capture of the eyes by new objects. Psychological Science, 9(5), 379-385. doi:10.1111/ 1467-9280.00071

Theeuwes, J., Kramer, A. F., Hahn, S., Irwin, D. E., \& Zelinsky, G. J. (1999). Influence of attentional capture on oculomotor control. Journal of Experimental Psychology: Human Perception and Performance, 25(6), 1595-1608. doi:10.1037/e536982012-682

Vatterott, D. B., \& Vecera, S. P. (2012). Experience-dependent attentional tuning of distractor rejection. Psychonomic Bulletin \& Review, 19(5), 871-878. doi:10.3758/s13423-012-0280-4 